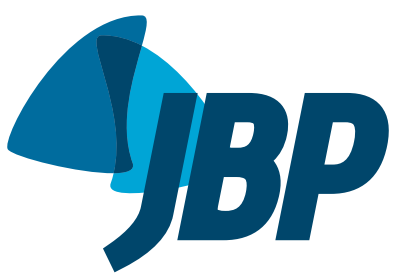

\section{Solitary fibrous tumor of the pleura: a rare cause of elevation of the right lung base}

\author{
Arthur Soares Souza Jr, ${ }^{1,2, a}$, Luciana Volpon Soares Souza ${ }^{1, b}$, \\ Gláucia Zanetti ${ }^{3, c}$, Edson Marchiori ${ }^{3, \mathrm{~d}}$
}

\section{DEAR EDITOR:}

A 42-year-old female nonsmoker with no history of comorbidities was admitted to the hospital with a 6-month history of right-sided chest pain and dyspnea. A chest X-ray showed marked elevation of the right lung base (Figures $1 \mathrm{~A}$ and $1 \mathrm{~B})$. Laboratory test results were unremarkable. A magnetic resonance imaging scan of the chest revealed a heterogeneous mass in the right hemithorax, with intermediate signal intensity on T1- and T2-weighted images (Figures 1C and 1D). A transthoracic needle biopsy was performed, and the results were inconclusive. The patient underwent radical surgical resection by open thoracotomy. The tumor was pedunculated, with free margins, measuring approximately $12 \times 11 \times 6 \mathrm{~cm}$, and the pedicle was connected to the mediastinal pleura. The final diagnosis was solitary fibrous tumor of the pleura (SFTP). The postoperative evolution was uneventful.

Elevation of the right lung base may occur associated with elevation of the right hemidiaphragm or with the diaphragm in normal position. In the first condition, the main causes are phrenic paralysis ${ }^{(1)}$ and the presence of an expansive lesion (e.g., a hepatic tumor or a subphrenic abscess) below the diaphragm. In the second condition, the main cause is infrapulmonary pleural effusion, although other, uncommon, causes include pleural tumors, such as SFTP.

A solitary fibrous tumor is defined as a mesenchymal neoplasm that has fibroblastic characteristics and clear peripheral vascular tumor-like branching vascularization. Although such tumors most commonly affect the pleura, they can occur in other thoracic areas (i.e., the mediastinum, pericardium, and lung), as well as in extrathoracic areas (i.e., the abdomen, head/neck, and central nervous system). ${ }^{(2-4)}$

An SFTP is often asymptomatic and discovered incidentally by radiography performed for other reasons. When signs and symptoms (including digital clubbing and hypertrophic osteoarthropathy) are present, they are usually associated with larger tumors. Patients with SFTP occasionally have hypoglycemia, which is seen more often in patients with malignant SFTP and is known as DoegePotter syndrome. Doege-Potter syndrome is believed to be a type of non-insulin-dependent hypoglycemia. ${ }^{(2,3)}$

Computed tomography (CT) of a small SFTP frequently demonstrates a homogeneous, well-defined, noninvasive, lobular soft-tissue mass, usually adjacent to the chest wall or within a fissure. Larger lesions are typically heterogeneous and may not exhibit CT features suggestive of focal pleural tumors. ${ }^{(4)}$ Heterogeneous areas of low attenuation on unenhanced CT scans may be caused by hemorrhage, necrosis, or cystic changes. ${ }^{(3,4)}$ Changes in tumor location can be detected and are often related to the attachment of a benign SFTP to the pleural tissue through the pedicle. ${ }^{(3)}$ Most localized fibrous tumors arise from the visceral pleura, and nearly half are pedunculated, the vascular supply to the tumor being contained within the pedicle. ${ }^{(4)}$

SFTPs show variable signal intensity on magnetic resonance imaging. The masses have predominantly low or intermediate signal intensity on T1- and T2-weighted images. They may also present high signal intensity on T2-weighted images. It has been suggested that this variable signal intensity is mainly dependent on the relative amounts of collagen and fibroblasts, as well as on the presence of areas of hemorrhage, necrosis, or cystic degeneration within the tumor. Intense heterogeneous enhancement after intravenous administration of gadolinium is typical and is generally due to high vascularity.

Making the differential diagnosis between benign and malignant SFTP is usually problematic. Although some imaging aspects, such as changes in the tumor location (suggesting the presence of a pedicle) and homogeneous attenuation of the lesion, are often associated with benign tumors, most authors have found that malignant lesions are indistinguishable from those with benign histological characteristics on imaging methods. ${ }^{(2,3-5)}$ Currently, SFTP is primarily diagnosed on the basis of microscopic pathology findings, especially those obtained with immunohistochemical techniques. ${ }^{(2,4)}$ On histological analysis, localized fibrous tumors appear as low-grade neoplasms with variable cellularity. The tumor cells are ovoid to spindle-shaped with round to oval nuclei, evenly distributed fine chromatin, inconspicuous nucleoli, and bipolar faintly eosinophilic cytoplasm with indistinct cell borders. Nuclear pleomorphism is minimal, and mitoses are usually rare or absent. Cellularity is variable and is inversely related to collagen content. Areas of necrosis, hemorrhage, or cystic degeneration may be evident, particularly in lesions that are large or in malignant lesions. Immunohistochemical staining shows that tumor cells are immunoreactive for CD34 and $\mathrm{Bcl}-2$, albeit they typically lack expression for cytokeratin or S-100 protein. ${ }^{(2-4)}$ Complete surgical excision is the treatment of choice and is the only effective treatment. 


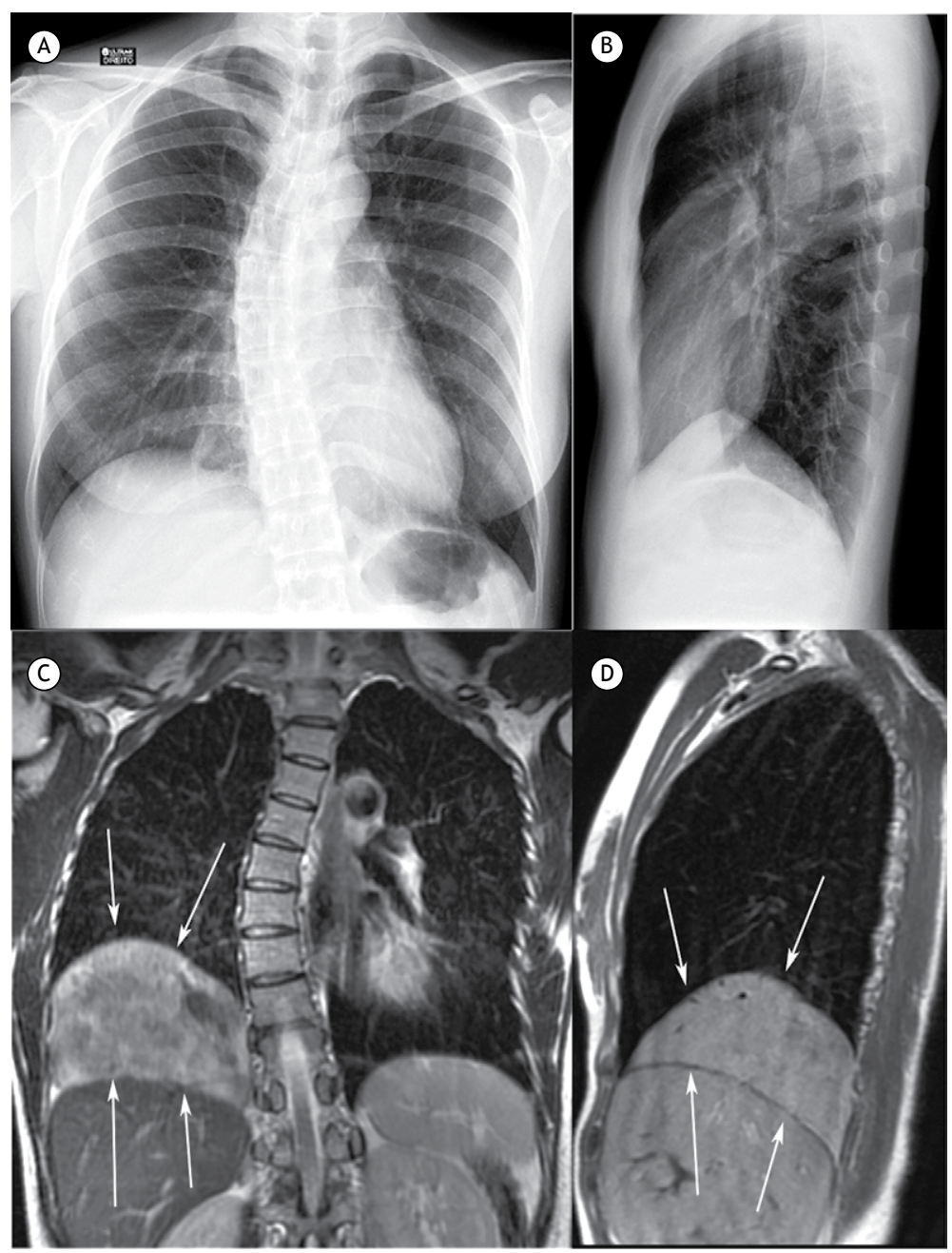

Figure 1. Frontal and lateral chest $X$-rays ( $A$ and $B$, respectively) showing elevation of the right lung base. Unenhanced coronal T2-weighted and sagittal T1-weighted magnetic resonance imaging scans ( $C$ and $D$, respectively) showing a large mass (arrows) occupying the inferior region of the right hemithorax with a heterogeneous, intermediate signal. The mass lies between the right lung base and the liver, with a well-defined cleavage plane between the two.

The prognosis for patients with SFTP is generally favorable. However, in a small number of cases, the lesions recur, undergo malignant transformation, or metastasize. $(2,3)$

\section{REFERENCES}

1. Ko MA, Darling GE. Acquired paralysis of the diaphragm. Thorac Surg Clin. 2009;19(4):501-10. https://doi.org/10.1016/] thorsurg.2009.08.011

2. Chu X, Zhang L, Xue Z, Ren Z, Sun YE, Wang M, et al. Solitary fibrous tumor of the pleura: An analysis of forty patients. J Thorac Dis. 2012;4(2):146-54. https://doi.org/10.3978/j.issn.20721439.2012.01.05

3. Rosado-de-Christenson ML, Abbott GF, McAdams HP, Franks TJ, Galvin JR. From the archives of the AFIP: Localized fibrous tumor of the pleura. Radiographics. 2003:23(3):759-83. https://doi. org/10.1148/rg.233025165

4. Cardinale L, Ardissone F, Garetto I, Marci V, Volpicelli G, Solitro F, et al. Imaging of benign solitary fibrous tumor of the pleura: a pictoria essay. Rare Tumors. 2010;2(1):e1. https://doi.org/10.4081/rt.2010.e1

5. Lococo F, Cafarotti S, Treglia G. Is 18F-FDG-PET/CT really able to differentiate between malignant and benign solitary fibrous tumor of the pleura? Clin Imaging. 2013;37(5):976. https://doi.org/10.1016/j. clinimag.2013.03.003 